Website: http://revistas.lamolina.edu.pe/index.php/acu/index

(C) Universidad Nacional Agraria La Molina, Lima - Perú

\title{
Brecha del servicio de limpieza pública en la ciudad de Tingo Maria, Perú
}

\author{
Gap of the public cleaning service in the city of Tingo Maria, Peru
}

\author{
Franklin Dionisio Montalvo
}

\begin{abstract}
Resumen
El objetivo de este estudio fue determinar el porcentaje de aceptación del servicio de limpieza pública y el nivel de servicio deseado en la ciudad de Tingo María, en el año 2016. Para ello, se realizó encuestas. Los resultados obtenidos muestran que la población desaprueba el nivel de equipamiento y unidades móviles del sistema de recolección de residuos sólidos, tipo de infraestructura de manejo y tratamiento de residuos sólidos, nivel de cumplimiento de responsabilidades adquiridas por el área de limpieza pública, nivel de interés en solucionar el problema del usuario, la ejecución del servicio según responsabilidad adquirida, el énfasis en registrar los errores, la exactitud en la comunicación de la realización de los servicios, disposición a ayudar de los trabajadores, disposición de los trabajadores a responder preguntas, manejo de residuos sólidos. Finalmente la brecha del servicio de limpieza pública en la ciudad de Tingo María aún es amplia, debido a que el $68 \%$ de los criterios consultados carecen de aprobación y requieren de mayor énfasis en fortalecer los conocimientos de los trabajadores para mejorar la atención, renovar unidades móviles, mejorar la infraestructura de tratamiento de residuos sólidos y construir un relleno sanitario con la finalidad de mejorar la calidad del servicio de limpieza pública.
\end{abstract}

Palabras clave: Servicio de limpieza pública; Brecha; Residuos sólidos; SERVQUAL.

\begin{abstract}
The objective of this study was to determine the percentage of acceptance of the public cleaning service and the level of service desired in the city of Tingo María, in 2016. For this purpose, surveys were carried out. The results obtained show that the population disapproves of the level of equipment and mobile units of the solid waste collection system, type of solid waste management and treatment infrastructure, level of compliance with responsibilities acquired by the public cleaning area, level of interest in solving the user's problem, the execution of the service according to acquired responsibility, the emphasis on recording errors, the accuracy in communicating the performance of services, willingness to help workers, willingness of workers to answer questions, management of solid waste. Finally, the gap in the public cleaning service in the city of Tingo María is still wide, because $68 \%$ of the criteria consulted lack approval and require greater emphasis on strengthening the knowledge of workers to improve care, renovate units mobile, improve the infrastructure for the treatment of solid waste and build a sanitary landfill in order to improve the quality of the public cleaning service.
\end{abstract}

Keywords: Public cleaning service; breach; solid residues; SERVQUAL.

\section{Introducción}

La gestión de residuos sólidos municipales por mucho tiempo ha sido una actividad olvidada dentro de la gestión pública; por ello, el VI Informe nacional de residuos sólidos municipales y no municipales del Perú (2014), asevera que solo el 1,62\% (304 t/d) de los residuos sólidos municipales son recogidos mediante el programa de segregación en la fuente y de estos el 0,3\% (63 t/d) de los residuos sólidos producidos a nivel nacional pasan por el proceso de compostaje. Esta condición es similar en la región Huánuco; por ello, SIGERSOL (2015), sostiene que solo el 11\% (ocho distritos) de los gobiernos locales de la región cuentan con el programa de segregación en la fuente y de ellos solo seis municipalidades brinda el servicio de tratamiento de residuos sólidos, siendo la Municipalidad
Provincial de Leoncio Prado uno de ellos. Sin embargo Nuñez (2016) afirma que para el caso del gerenciamiento de residuos sólidos, existe la necesidad de implementar rellenos, pero sería una solución inmediata, se debe hacer un plan de manejo estratégico, y someterlo a consideración y aportes de la comunidad.

Los análisis realizados por Vargas (2011), al agua, aire y suelo determinaron presencia de microorganismos en residuos sólidos y líquidos en el botadero "La Muyuna" indicando un nivel bajo de calidad ambiental. "La Muyuna" es un botadero de más de 30 años de antigüedad, ubicado en la ciudad de Tingo María, provincia de Leoncio Prado, departamento de Huánuco (OEFA, 2014a). Recibía hasta 35 toneladas de basura al día, incluyendo residuos hospitalarios, que eran vertidos al río Huallaga. Desde el 
año 2012, el OEFA viene recomendando a la Municipalidad Provincial de Leoncio Prado priorizar la ejecución del proyecto de implementación de un relleno sanitario, implementar en el menor plazo posible un plan de cierre, y ejecutar un programa de formalización de recicladores, por ello, reiteró a dicha municipalidad que cumpla con las recomendaciones formuladas frente a la problemática del botadero "La Muyuna" (OEFA, 2014b). En consecuencia, es importante conocer el nivel de aprobación del servicio de limpieza pública de parte de la población, por ello en la presente investigación se analiza la percepción con respecto al servicio de limpieza pública medido en el ámbito de la zona urbana de la ciudad de Tingo María.

\section{Materiales y métodos}

La investigación se realizó en la ciudad de Tingo María, cuyas coordenadas UTM son 18L 0390326,8970861, ubicado en el distrito de Rupa Rupa, provincia de Leoncio Prado, región Huánuco, durante los meses de febrero a julio del 2016.

El formato de encuestas se estructuró buscando conocer la percepción de la población sobre la implementación del servicio de limpieza pública en la Provincia de Leoncio Prado en la situación actual y deseada.

Supo (2013) afirma que en una encuesta con escala sus alternativas tendrán un carácter ordinal, asimismo el mismo autor manifiesta que si queremos evaluar las actitudes, conductas u opiniones, una escala será el instrumento más adecuado. Wigodski (2003) afirma que SERVQUAL es conocido como "Modelo de discrepancias" y sugiere que la diferencia entre las expectativas generales de los clientes $\mathrm{y}$ sus percepciones respecto al servicio de un proveedor específico pueden constituir una medida de la calidad en el servicio. Por ello, para la realización de encuestas con escalas se empleó el modelo SERVQUAL adaptado.

Para determinar el número de muestras, se debe conocer los valores de "p" y "q" por ello se realizó una encuesta piloto donde se planteó en forma directa la realización de 20 encuestas, para lo cual se identificaron las viviendas respectivas en forma aleatoria, procediendo luego a realizar la presentación del tema de investigación, una inducción rápida y la aplicación de las encuestas al representante de la vivienda, con ello se pudo realizar una primera aproximación sobre la percepción de la población sobre la aceptación del servicio de limpieza pública. De la encuesta Piloto se obtuvo los siguientes valores:

$\mathrm{p}=$ Probabilidad de éxito (aprobación) 0,5\%

$\mathrm{q}=$ Probabilidad de fracaso (desaprobación) 0,5\%

Webster (2000), desarrolló la fórmula de Muestreo Aleatorio Simple (MAS) con población finita (muestreo sin reemplazo), tomando en cuenta el tamaño poblacional:

Dónde:

$$
n=\left(\mathrm{pqz}^{2} \mathrm{~N}\right) /\left((\mathrm{N}-1) \mathrm{e}^{2}+\mathrm{pqz}^{2}\right)
$$

$\mathrm{n}=$ Muestra de las viviendas.

$\mathrm{N}=$ Total de viviendas $=(58698 \mathrm{hab} / 5)=11739$ viviendas
$\mathrm{Z}=$ Nivel de confianza $95 \%=1,96$

$\mathrm{e}=$ Error permisible $(0,05)$.

$\mathrm{p}=$ Probabilidad de éxito $=0,5 \%$;

$\mathrm{q}=$ Probabilidad de fracaso $=0,5 \%$;

A partir de los valores obtenidos para "p" y "q" en la encuesta piloto (en porcentajes donde $\mathrm{p}+\mathrm{q}=100 \%$ ) y empleando la formula anterior se calculó que el número total de muestras fuera 95, posterior a ello se identificó aleatoriamente nuevas viviendas seleccionadas y se procedió a realizar la presentación del tema de investigación, una inducción rápida y la aplicación de las encuestas al representante de la vivienda, con ello se pudo conocer la percepción de la población con respecto al nivel del servicio de limpieza pública que recibe.

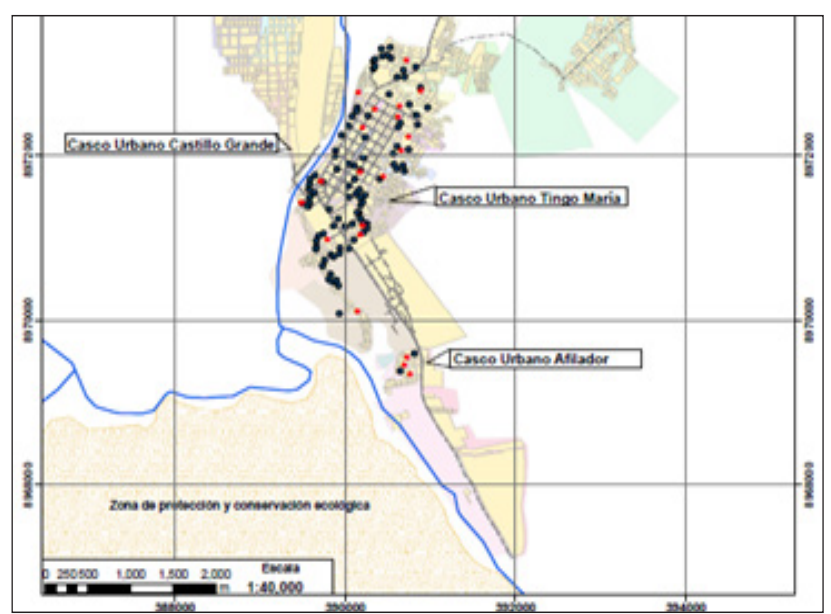

Figura 1. Ubicación espacial de la distribución de las encuestas

Con la información obtenida mediante la encuesta definitiva se procedió a validar los datos con el Alpha de Cronbach, obteniendo valores de 0,$888 ; 0,863$ y 0,886 para la encuesta piloto, encuesta definitiva en la situación actual y encuesta definitiva en la situación deseada quedando validados los resultados obtenidos. Posteriormente se procedió a generar la tendencia de la percepción de la situación actual y la situación deseada del servicio de limpieza pública, determinando finalmente la brecha del servicio.

\section{Resultados y discusión}

La percepción de la población de la ciudad de Tingo María con respecto al servicio de limpieza pública que brinda la Municipalidad Provincial de Leoncio Prado, son mostrados a continuación.

De la Figura 2 ítems a y b se desprende que la población de la ciudad de Tingo María desaprueba el estado actual del equipamiento y las unidades móviles de recolección de residuos sólidos, asimismo desaprueba también las características de la infraestructura de manejo y tratamiento de residuos sólidos por estar construida con material rústico. 
Gonzales y Huamán (2015) realizó el diagnóstico situacional de la gestión integral de residuos sólidos del distrito de Contumazá describiendo la gestión como "tradicional", agrega también que la cobertura del servicio de limpieza pública en esa ciudad es ineficiente. En los ítems e y f de la Figura 2 se observa también que la población de la ciudad de Tingo María desaprueba el nivel de cumplimiento de responsabilidades adquiridas por el área de Limpieza Pública y resalta la desaprobación del interés mostrado por los trabajadores en solucionar el problema del usuario. Rentería y Zeballos (2014) manifiestan que satisfacer al ciudadano del distrito de los Olivos es una tarea compleja, se necesita no solo el Programa se adapte a él, sino también que el mismo vecino necesite cambiar sus costumbres para lograr un objetivo en común.

Maguiña (2014) afirma que el nivel de eficiencia del servicio público de limpieza afecta de manera negativa en la satisfacción de los usuarios de la zona urbana de la Municipalidad Distrital de Independencia. En la Figura 3 de nuestra investigación se observa el nivel de aprobación de la población de la ciudad de Tingo María con respecto a la presentación personal de los trabajadores del área de limpieza pública, en segundo lugar la calidad del material empleado para las actividades de comunicación visual, finalmente la aprobación a la rapidez y eficacia de realización del servicio de limpieza pública. Ayma y Peñafiel (2011) afirman que la cobertura de recolección en el distrito de Chinchero tiene una eficiencia de 0,376 , siendo menor a 0,7 (nivel óptimo), calificado por ellos como poco aceptable. En nuestro caso se resalta la aprobación de la rapidez con la que se realiza el servicio de recolección de residuos sólidos (Aspecto visible a la ciudadanía).

Gonzales y Huamán (2015) manifiestan que las principales deficiencias y necesidades de los sistemas de manejo de la gestión de residuos sólidos del distrito de Contumaza - Cajamarca son los escasos recursos económicos destinados al servicio de limpieza pública. Sin embargo existen aspectos como el cumplimiento de responsabilidades adquiridas (Compromisos) o la disponibilidad a ayudar de parte de los trabajadores o aspectos como los hábitos para el registro de errores o la exactitud en la comunicación de realización del servicio que no necesariamente requieren de recursos económicos para lograr su cumplimiento por lo que la población de la ciudad de Tingo María desaprueba los aspectos mencionados en la Figura 4.

Según los resultados mostrados en la Figura 5 se obtiene que la población de la ciudad de Tingo María desaprueba el manejo de residuos sólidos realizado hasta el año 2016 complementando con la desaprobación de la disponibilidad de los trabajadores a responder las preguntas de turno producto de una atención individualizada que busca recibir la población, es por ello que la población considera que los trabajadores no cuentan con los conocimientos necesarios para el desarrollo del servicio. Rentería y Zeballos (2014) manifiestan que los procesos estratégicos del Programa de Segregación en la Fuente y Recolección Selectiva de Residuos Sólidos Domiciliarios del distrito de Los Olivos es inadecuado y se evidencia en las decisiones poco planificadas y no orientadas hacia el buen uso de recursos, pues los costos operativos están creciendo significativamente año tras año.
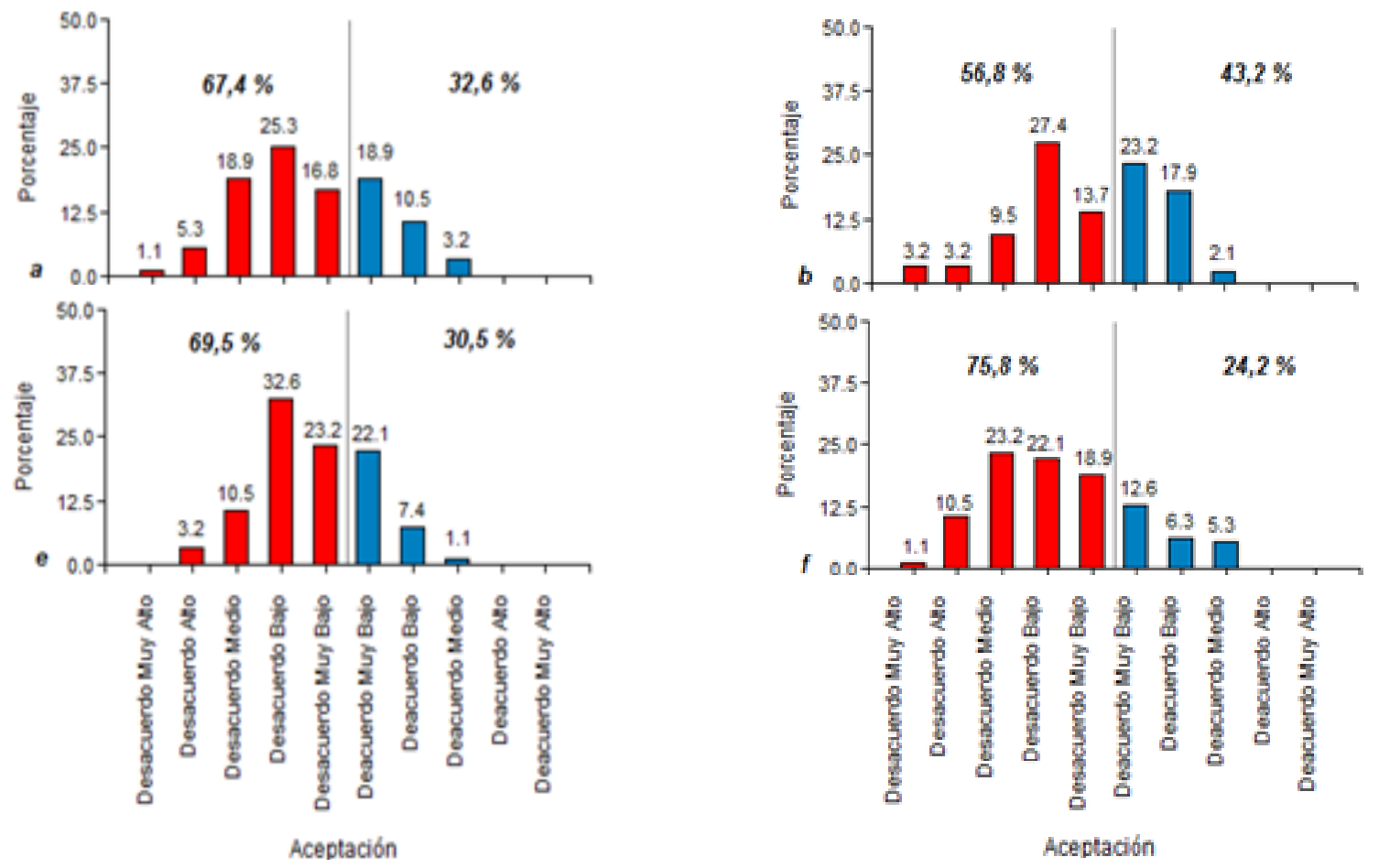

Figura 2. a) Equipamiento y unidades móviles de recolección de residuos sólidos; b) Infraestructura de manejo y tratamiento de residuos sólidos; e) Cumplimiento de responsabilidades adquiridas; f) Interés en solucionar el problema del usuario. 

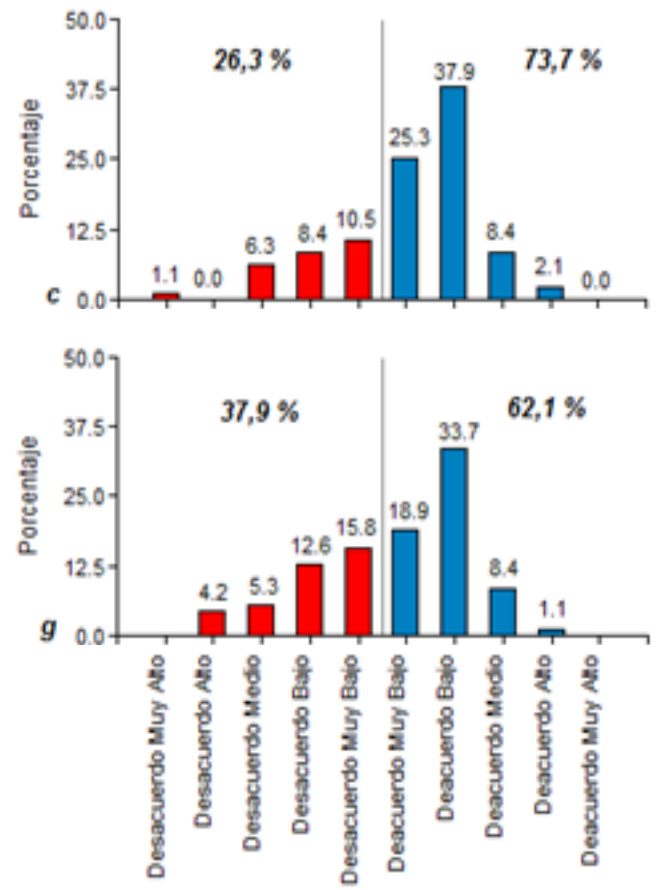

Aceptación
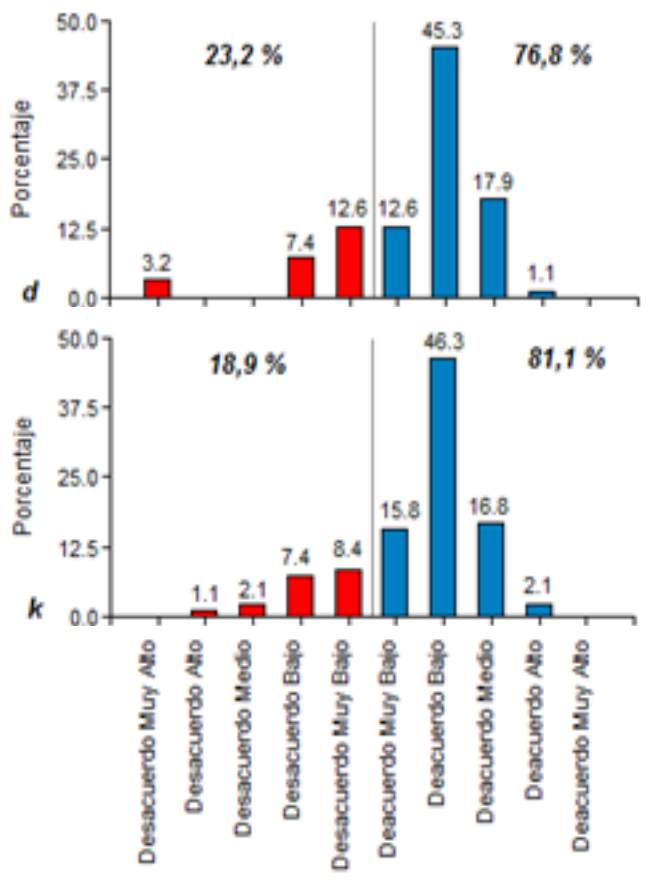

Aceptación

Figura 3. c) Presentación personal de los trabajadores; d) Calidad del material de comunicación visual; g) Eficacia en la realización del servicio; k) Rapidez de la realización del servicio.

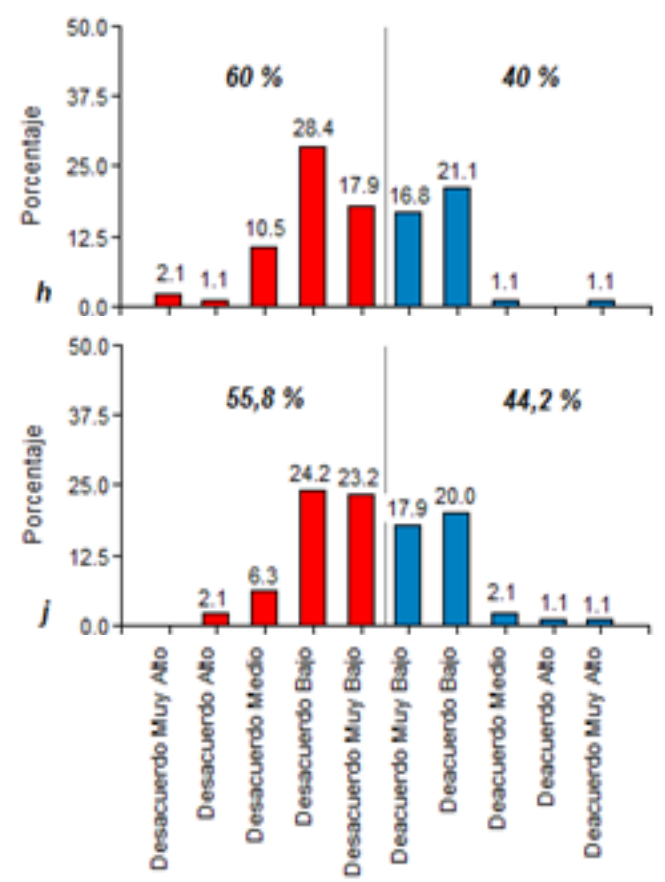

Aceptación
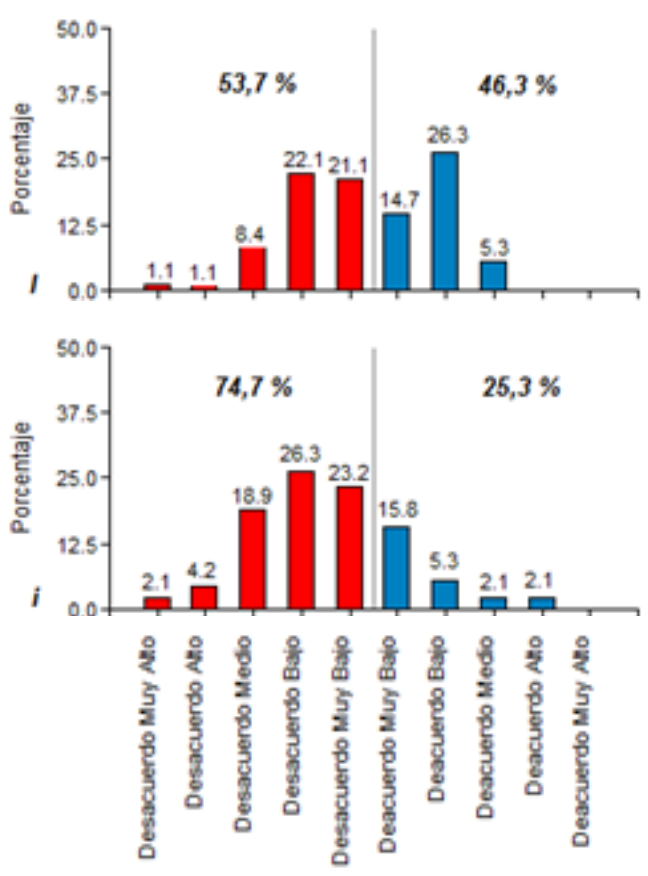

Aceptación

Figura 4. h) Cumplimiento de responsabilidades adquiridas; i) Registro de errores; j) Exactitud de la comunicación de la realización del servicio; 1) Disponibilidad a ayudar de parte de los trabajadores. 

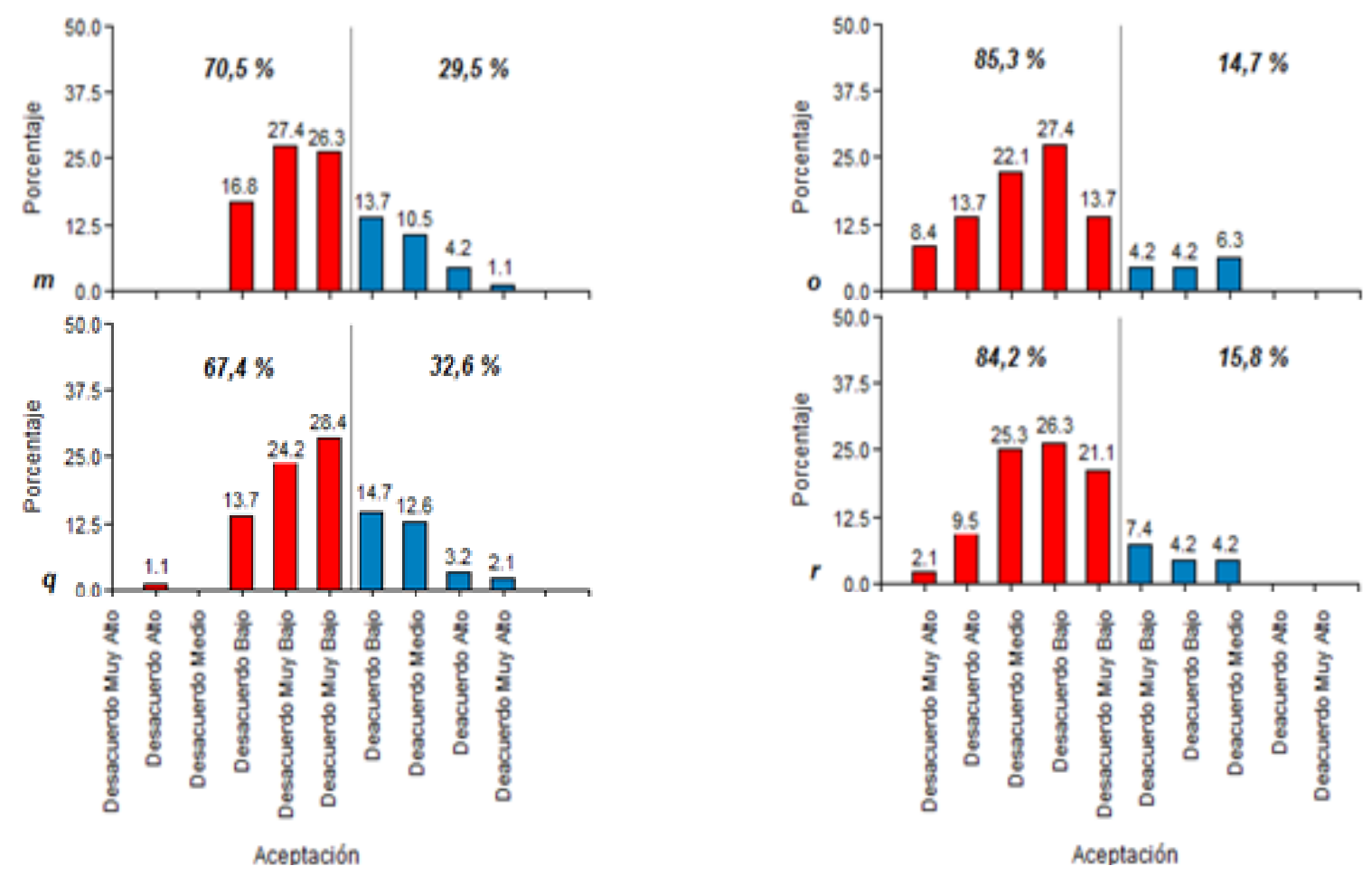

Figura 5. m) Disponibilidad a responder de parte de los trabajadores; o) Manejo de residuos sólidos; q) Conocimientos necesarios de los trabajadores; r) Atención individualizada de parte de la Municipalidad.
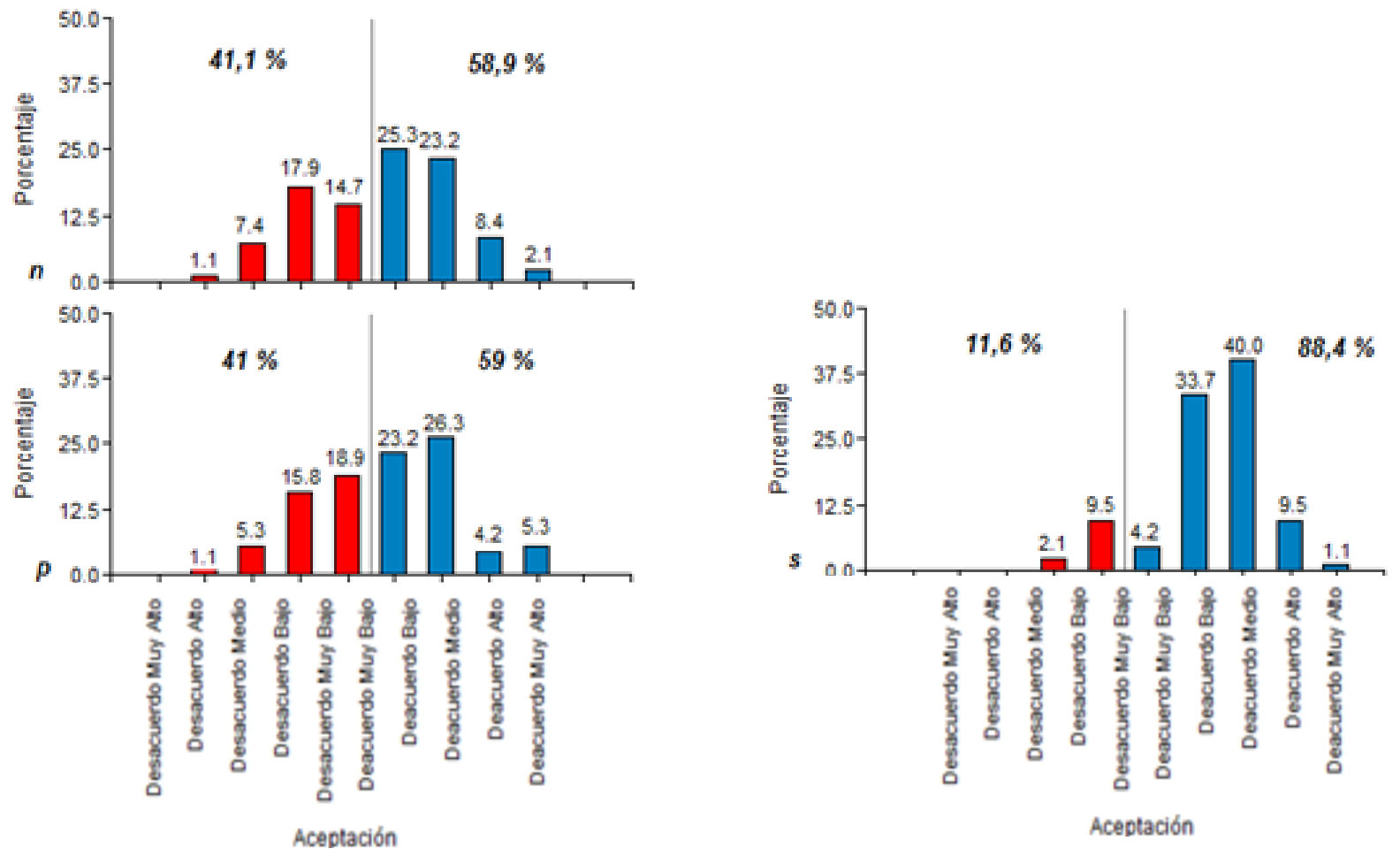

Figura 6. n) El comportamiento de los trabajadores inspira confianza; p) Demostración de cortesía de parte de los trabajadores del área de limpieza pública; s) El área tiene horarios adecuados para todos sus beneficiarios. 
La Figura 6 muestra que la población de la ciudad de Tingo María con porcentajes de 58,9\% los aspectos de comportamiento y demostración de cortesía de parte de los trabajadores de la Municipalidad Provincial de Leoncio Prado, se agrega también la aceptación del horario establecido para el servicio de recolección de residuos sólidos. Cabe precisar que en la Figura 5 se aprecia que la misma población desaprueba aspectos relacionados al conocimiento de los trabajadores con respecto al servicio, mostrando criterios diferentes de evaluación de parte de la ciudadanía.

Maguiña (2014) manifiesta que el nivel de eficiencia del servicio público de limpieza en el Distrito de Independencia fue calificado según la percepción del cliente como regular. Según los resultados mostrados en la Figura 7 se evidencia que la población de la ciudad de Tingo María requiere de atención personalizada y servicio de buena calidad.

Wigodski (2003) afirma que SERVQUAL es conocido como "Modelo de discrepancias" y sugiere que la amplia labor que debe desarrollar la Municipalidad Provincial de Leoncio Prado.

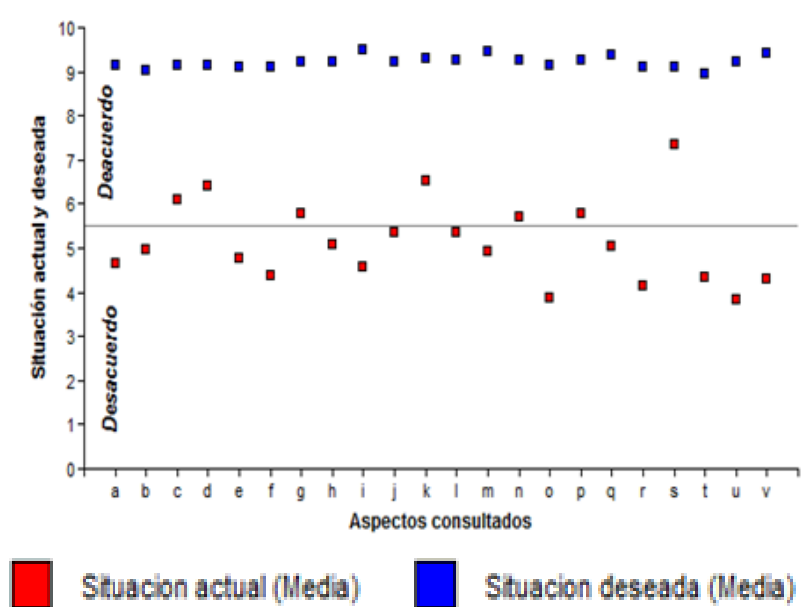

Figura 8. Brecha del servicio de limpieza pública a nivel de la percepción de la población
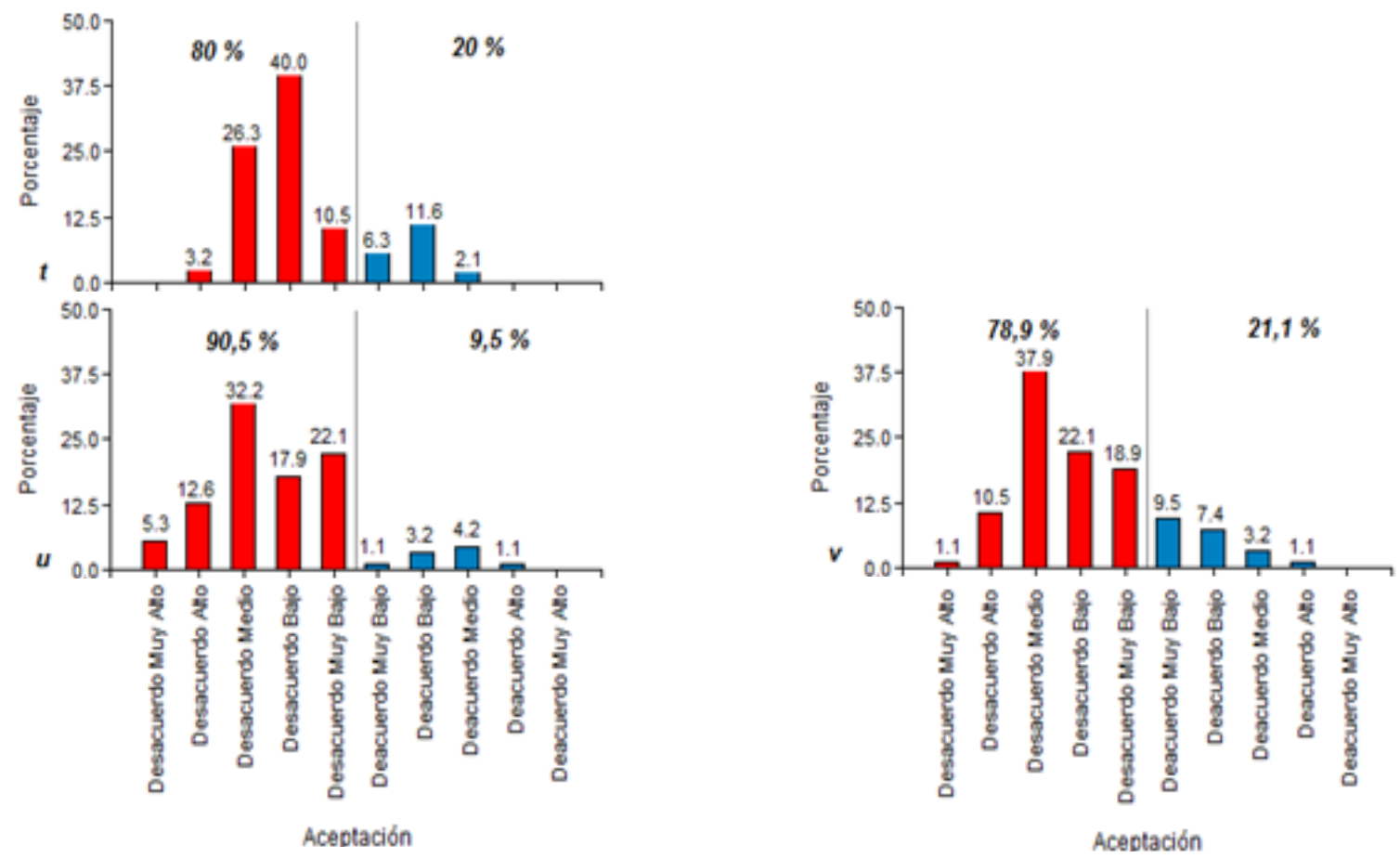

Figura 7. t) Atención personalizada de los trabajadores; u) Interés por brindar un mejor servicio de limpieza pública; v) Comprensión de los trabajadores de las necesidades específicas de los usuarios del servicio.

la diferencia entre las expectativas generales de los clientes y sus percepciones respecto al servicio de un proveedor específico pueden constituir una medida de la calidad en el servicio. En nuestra investigación se realizó una adaptación del formato de encuesta SERVQUAL para evaluar el nivel de satisfacción del servicio de limpieza pública, los resultados se aprecian en la Figura 8 donde la mayoría de los aspectos consultados a la población muestran un desacuerdo en el nivel de implementación, asimismo se consultó el nivel deseado cuyo diferencial representa

\section{Conclusiones}

La percepción de la población de la ciudad de Tingo María muestra su desaprobación del servicio en 15 de 22 aspectos; siendo la calidad de las unidades de transporte, tipo de infraestructura para tratamiento de residuos sólidos, cumplimiento de compromisos adquiridos, interés mostrado en solucionar problemas del usuario, exactitud en la comunicación, conocimientos necesarios de parte de los trabajadores para responder preguntas, ayudar a los usuarios y mostrar interés en brindar un mejor 
servicio identificando las necesidades específicas como parte de la atención personalizada que debe brindar la Municipalidad Provincial de Leoncio Prado, asimismo la población aprobó siete de 22 aspectos consultados tales como la rápidez y capacidad de ejecución del servicio en la primera oportunidad, buena presencia, reflejo de confianza y demostración de cortesía de los trabajadores del área de limpieza pública, así como el horario establecido para la recolección y la calidad de los materiales empleados en la difusión del servicio de limpieza pública. Finalmente la brecha del servicio de limpieza pública en la ciudad de Tingo María aún es amplia, lo que significa que la institución responsable debe enfatizar en fortalecer los conocimientos de los trabajadores para mejorar la atención, renovar unidades móviles, mejorar la infraestructura de tratamiento de residuos sólidos y construir un relleno sanitario con la finalidad de mejorar la calidad del servicio de limpieza pública.

\section{Literatura citada}

Ayma, M.L. y Peñafiel, T. 2011. Diagnóstico y propuesta de plan de manejo ecoeficiente de los residuos sólidos en el centro poblado del distrito de Chinchero. Conclusiones. Tesis Ingeniero Agrónomo, Universidad Nacional San Antonio Abad del Cusco, Cusco, Perú. $207 p$.

Gonzales, D.C; Huamán, G.N. 2015. Gestión de residuos sólidos urbanos en el distrito de Contumazá, provincia de Contumazá, Cajamarca. Conclusiones. Tesis Ingeniero Industrial, Universidad Nacional de Trujillo, Trujillo, Perú. 226p.

Maguiña, M.P. 2014. La eficiencia del servicio público de limpieza y la satisfacción de los usuarios de la municipalidad del distrito de independencia. Conclusiones. Tesis Magister Scientiae, Administración Mención en Administración de Negocios. Universidad Nacional Santiago Antúnez de Mayolo, Huaraz, Perú. $162 \mathrm{p}$.

Nuñez, H. 2016. Estado actual de la gestión ambiental del Perú. Lima, Perú. 13p.

OEFAa, 2014. Fiscalización eficiente. Caretas, Lima. s.v.nr. 1-12p.

OEFAb, 2014. Boletín Informativo $\mathrm{N}^{\circ}$ 023. Lima. s.v.nr. $1-17 \mathrm{p}$.

Rentería, J.M y Zeballos, M.E. 2014. Propuesta de mejora para la gestión estratégica del Programa de Segregación en la Fuente y Recolección Selectiva de Residuos Sólidos Domiciliarios en el distrito de Los Olivos. Tesis Licicenciado, Gestión con mención Gestión Empresarial, Pontífice Universidad Católica del Perú. Lima, Perú. 213p.

SIGERSOL, 2015. Sistema de información para la gestión de residuos sólidos. SIGERSOL. Disponible en: http:// www.sigersol.gob.pe, Informes, 05 Set. 2015.

Supo, J. 2013. Como validar un instrumento. Bioestadístico. Disponible en: http://www.bioestadistico.com,
Informes, 7 Abr. 2016.

Vargas, K. 2011. Indicadores microbiológicos de calidad ambiental del botadero La Moyuna. Conclusiones. Informe de Practicas Pre Profesionales Bach. Ciencias Ambientales. Tingo María, Perú. 85p.

VI informe nacional de residuos sólidos municipales y no municipales, 2014. Residuos sólidos municipales y no municipales. Lima, Perú. 4-5p.

Webster, A.L. 2000. Estadística aplicada a los negocios y la economía. Mc Graw Hill. 3 ed. México DF, México. $651 \mathrm{p}$.

Wigodski, J. 2003. ¿Qué es SERVQUAL?. Medwave. Nov 10p. Disponible en: https://www.medware.cl/link.cgi/ Medware/Enfermeria/Nov2003/2763. 